\title{
BMJ Open Hospital-diagnosed atopic dermatitis and long-term risk of myocardial infarction: a population-based follow-up study
}

Jette Lindorff Riis, ${ }^{1}$ Christian Vestergaard, ${ }^{1}$ Kasper Fjellhaugen Hjuler, ${ }^{1}$ Lars Iversen, ${ }^{1}$ Lars Jakobsen, ${ }^{2}$ Mette S Deleuran, ${ }^{1}$ Morten Olsen ${ }^{3}$

To cite: Riis JL,

Vestergaard C, Hjuler KF, et al. Hospital-diagnosed atopic dermatitis and longterm risk of myocardial infarction: a population-based follow-up study. BMJ Open 2016;6:e 011870. doi:10.1136/bmjopen-2016011870

- Prepublication history and additional material is available. To view please visit the journal (http://dx.doi.org/ 10.1136/bmjopen-2016011870).

Received 11 March 2016 Revised 3 August 2016 Accepted 9 September 2016

CrossMark

For numbered affiliations see end of article.

Correspondence to Dr Jette Lindorff Riis; jette_riis@hotmail.com

\section{ABSTRACT}

Objective: Atopic dermatitis (AD) is an inflammatory skin disorder with a childhood prevalence reaching $20 \%$. An estimated $50 \%$ of patients have a life-long chronic course. The purpose of this study was to estimate the risk of first-time myocardial infarction (MI) in patients with AD compared with a general population cohort.

Design: Cohort study.

Setting: Denmark.

Participants: Using population-based medical registries, we identified individuals born in Denmark from 1947 to 1983 with at least two hospital-diagnoses of AD following inpatient admissions or hospital-based outpatient visits at any age from 1977 to 2013. Individuals with $A D$ were matched with general population controls (10:1) for birth-year and gender. Unique personal identifiers permitted unambiguous data linkage.

Primary outcome measures: Follow-up began on the date of $A D$ diagnosis (index date for general population controls) and continued until death, emigration, $\mathrm{MI}$ or the year 2013. We computed the 15-year-cumulative incidence of $\mathrm{MI}$ following a diagnosis of $\mathrm{AD}$. Comparing patients with $A D$ with the general population cohort, we computed HRs of MI presented with $95 \%$ Cls and adjusted for history of diabetes mellitus, hypertension, hyperlipidaemia or stroke, educational level, birth-year and sex.

Results: We identified 4814 patients diagnosed with $A D$. The cumulative incidence of MI was $0.6 \%$ for patients with $A D$ and $0.4 \%$ for their matched controls. The corresponding adjusted HR was 1.74 (1.21 to 2.49). The HR for patients who were not in need of systemic treatment was 1.58 (1.02 to 2.45) and it was 2.40 (1.27 to 4.45$)$ for those who were treated with azathioprine, methotrexate or cyclosporine.

Conclusions: Hospital-diagnosed AD was associated with increased risk of $\mathrm{MI}$ compared with the general population.

\section{INTRODUCTION}

Atopic dermatitis (AD) is a chronic, or chronically relapsing, thymus-derived helper

\section{Strengths and limitations of this study}

- Strengths include the population-based design and large sample size, with long-term follow-up for more than 30 years from diagnosis of atopic dermatitis and the ability to adjust for multiple cardiovascular risk factors.

- Use of sensitivity analyses to investigate validity of atopic dermatitis diagnoses is another strength.

- We were limited by lacking data on smoking and body mass index.

- Despite the complete long-term follow-up and large sample size, subgroup analyses on patients diagnosed with atopic dermatitis before 20 years of age lacked precision.

cell type 2 (Th2) dominated inflammatory skin disease with severe barrier dysfunction. ${ }^{1}$ It is the most common chronic childhood disease. ${ }^{2}$ Disease severity varies from virtually negligible to substantially affecting quality of life. ${ }^{3}$

The childhood prevalence of $\mathrm{AD}$ has increased over the past decades, ${ }^{4}$ but now seems to have reached a plateau of $\sim 20 \%$ in affluent countries. ${ }^{5}$ A recent Danish study found persistent $\mathrm{AD}$ in adulthood in $50 \%$ of those diagnosed with AD at school age. ${ }^{6}$

Another common inflammatory skin disease, psoriasis, which is generally perceived to be a Th1/Th17 dominated disease, is known to be associated with an increased risk of myocardial infarction (MI). ${ }^{7}$ There are now indications that AD may also be associated with increased risk of MI. Studies have reported associations between childhood $\mathrm{AD}$ and being overweight, including increased central obesity, as well as high blood pressure. 8 Recently, we reported an increased prevalence of asymptomatic coronary artery disease among individuals with AD, assessed 
by cardiac CT angiography, compared with non-AD control individuals. ${ }^{9}$ Furthermore, studies have reported associations between $\mathrm{AD}$ in adults and history of selfreported heart attacks, ${ }^{10}$ cardiovascular risk factors, including smoking, diabetes mellitus and high serum cholesterol levels. ${ }^{11}$ However, a recent follow-up study reported no increased risk of $\mathrm{MI}$ in patients with $\mathrm{AD}$, after adjustment of diagnoses and indicators of cardiovascular risk factors. ${ }^{12}$

The purpose of the present study was to estimate the long-term risk of MI in individuals with hospitaldiagnosed $\mathrm{AD}$ compared with a birth-year-matched and gender-matched general population cohort.

\section{METHODS}

\section{Setting}

Our nationwide study was conducted in Denmark, which has a population of 5.6 million individuals. The Danish National Health Service provides tax-supported healthcare, with free access to hospital-based and primary medical care.

\section{Study cohorts and design}

We based this cohort study on data from populationbased registries. Using the Danish National Registry of Patients (DNRP) we identified all individuals born between 1 January 1947 and 1 January 1977 who received a hospital diagnosis of AD from 1 January 1977, when the DNRP was established, until 1 January 2013. The diagnoses were assigned following hospital admissions or outpatient clinic visits. Pre-existing MI was avoided by excluding patients and members of the comparison cohort with MI before AD diagnosis (index date for controls). The DNRP contains information on all hospital admissions in Denmark. Since 1995 data on outpatient visits were also included. It includes patient civil registration numbers, dates of admission (or outpatient clinic contact) and discharge, and up to 20 discharge diagnoses coded by the physicians according to the eighth edition of the International Classification of Diseases (ICD) from 1977 to 1993, and the 10th edition thereafter. The DNRP is widely used for research purposes, and the positive predictive value of the ICD diagnosis coding has been extensively studied and reported high for numerous diagnoses. ${ }^{13}$ There has been no studies of the positive predictive value of $\mathrm{AD}$ diagnoses specifically. However, we included only patients with at least two distinct inpatient or outpatient hospital contacts where the diagnosis of $\mathrm{AD}$ was assigned. For use in a sensitivity analysis, we identified a subgroup of patients with $\mathrm{AD}$, where $\mathrm{AD}$ was the primary diagnosis following at least two hospital contacts.

For each patient with $\mathrm{AD}$, we identified 10 individuals from the general population, matched for sex and birthyear, using the Danish Civil Registration System (CRS). The CRS has maintained electronic records on dates of birth, emigration and death for all Danish residents since $1968 .{ }^{14}$ Individuals are tracked with a unique personal identifier, which is assigned to every Danish citizen and used in all public Danish registries. The personal identifier permitted unambiguous individual-level linkage among the databases used in this study.

\section{Myocardial infarction}

We used the participants' personal identifiers in the patient cohort and the general population comparison cohort to search the DNRP for records on MI.

\section{Covariates}

Data on the educational level of patients and general population comparison cohort members were identified using Statistics Denmark. ${ }^{15}$ The highest educational level achieved was classified as primary education, secondary education or higher education. ${ }^{16}$ In the Danish National Prescription Registry established in $1995,{ }^{17}$ we identified the first date of filling a prescription for cyclosporine, azathioprine or methotrexate all used in Denmark for systemic $\mathrm{AD}$ treatment. In this registry, medications are classified according to the Anatomical Therapeutic Chemical (ATC) Classification. Using the DNRP, we identified patients and comparison cohort members with a history of MI risk factors in terms of diabetes mellitus, hypertension and hyperlipidaemia. Furthermore, we identified history of stroke as a proxy for these MI risk factors. See online supplementary appendix for the specific ICD and ATC codes used in this study.

The study was approved by the Danish Data Protection Agency whose role is to protect the privacy of individuals whose data are recorded in Danish registries. Participants were not required to give informed consent for this study.

\section{Data analysis}

Follow-up of patients with $\mathrm{AD}$ and the matched comparison cohort members began on the date of AD diagnosis (index date for patients in the comparison cohort). Follow-up continued until emigration, death, MI diagnosis or 1 January 2013, whichever occurred first. Cumulative MI incidence curves, with death as a competing risk, were computed for patients with $\mathrm{AD}$ and their respective matched comparison cohort members. ${ }^{18}$

We used Cox proportional hazards regression models to compute HRs of the time from $\mathrm{AD}$ diagnosis to $\mathrm{MI}$ compared with the general population cohort. The HRs were adjusted for gender, categories of birth-year (19471956, 1957-1966, 1967-1983) and educational level (short-term education, medium-term education, longterm education), as well as history of diabetes mellitus, hypertension, hyperlipidaemia or stroke. We did not have data on race or household income. Covariate selection was based on considerations concerning associations with $\mathrm{AD}$ and $\mathrm{MI}$ risk. As a substantial proportion of patients with $\mathrm{AD}$ may have had $\mathrm{AD}$ before being diagnosed at hospitals, we could not preclude that some of the covariates were in fact intermediate steps in a 
potential causal pathway from AD to MI. Adjustment for intermediate steps would lead us to underestimate the impact of $\mathrm{AD}$ on MI risk. We therefore also presented crude estimates, only controlling for birth-year and gender. Regression analyses comparing the $\mathrm{AD}$ cohort with the general population cohort were stratified by gender, year of birth categories and age at $\mathrm{AD}$ diagnosis. Additional regression models only included patients and comparison cohort members at risk of incident MI 1 January 1996 allowing for subgroup analyses based on covariates on drug use from the Danish National Prescription Database established in 1995. We identified indicators of the first dates of systemic $\mathrm{AD}$ treatment prescription filling. HRs adjusted for educational level, were based on patients with complete data. For all other variables on prescriptions and diagnoses, 'missing' data were interpreted as lacking occurrence of the given diagnosis or prescription filling. We verified graphically the assumption of proportional hazards. Analyses were performed with Stata 14 (StataCorp LP, College Station, Texas, USA).

\section{RESULTS}

\section{Characteristics}

We identified 4814 patients with $\mathrm{AD}$ with males accounting for $45 \%$. The educational level of patients with $\mathrm{AD}$ was comparable to that of the age-matched and gendermatched general population comparison cohort. Proportions with history of diabetes mellitus, hypertension, hyperlipidaemia or stroke were similar in the two cohorts. Of the 4639 patients with $\mathrm{AD}$ at risk of incident MI after 1 January 1996, 981 (21\%) filled at least one prescription for cyclosporine, azathioprine or methotrexate (table 1$)$.

\section{Myocardial infarction}

The median length of follow-up was 15.1 years in the AD cohort and 15.5 years in the matched general population cohort. The median age at the 35 MIs occurring in the $\mathrm{AD}$ cohort was 48.2 years and it was 48.5 years for the 203 MIs occurring in the general population cohort. The incidence rates of MI per 1000 person years in the two cohorts were 0.45 (95\% CI 0.32 to 0.62 ) and 0.25 (95\% CI 0.22 to 0.29 ), respectively. The cumulative incidence of MI after 15 years of follow-up from $\mathrm{AD}$ diagnosis was $0.6 \%$ in the overall $\mathrm{AD}$ cohort and $0.4 \%$ in the general population cohort (figure 1). The corresponding HR was 1.74 (95\% CI 1.21 to 2.49) after adjusting for year of birth, gender, educational level and a variable indicating history of diabetes, hypertension, hyperlipidaemia or stroke. We were able to account for prescriptions on systemic $\mathrm{AD}$ treatment during follow-up in the subcohort still at risk of MI after 1 January 1996. In this analysis, the overall crude HR was 1.83 (95\% CI 1.27 to 2.62) and after adjusting for the same variables as in the main analysis it was 1.79 (95\% CI 1.25 to 2.56). The adjusted HR comparing MI risk of patients with $\mathrm{AD}$ and
Table 1 Characteristics of 4814 patients diagnosed with atopic dermatitis in Denmark between 1977 and 2013 and a general population comparison cohort matched for birth-year and gender

\begin{tabular}{|c|c|c|}
\hline & $\begin{array}{l}\text { Atopic } \\
\text { dermatitis } \\
\text { Cohort } \\
\mathrm{n}(\%)\end{array}$ & $\begin{array}{l}\text { General } \\
\text { population } \\
\text { Cohort } \\
\text { n (\%) }\end{array}$ \\
\hline All & $4814(100)$ & $48396(100)$ \\
\hline Sex, male & $2144(45)$ & $21311(44)$ \\
\hline \multicolumn{3}{|l|}{ Birth year } \\
\hline 1947-1956 & $521(11)$ & $5141(11)$ \\
\hline 1957-1966 & $1104(23)$ & $11212(23)$ \\
\hline 1967-1983 & $3189(66)$ & $32043(66)$ \\
\hline \multicolumn{3}{|l|}{ Educational level } \\
\hline Short-term education & $1300(27)$ & $12434(26)$ \\
\hline $\begin{array}{l}\text { Medium-term } \\
\text { education }\end{array}$ & $1947(40)$ & $20923(43)$ \\
\hline Long-term education & $1485(31)$ & $14318(30)$ \\
\hline Missing & $82(2)$ & $720(1)$ \\
\hline Diabetes mellitus & $34(1)$ & $343(1)$ \\
\hline Hypertension & $52(1)$ & $381(1)$ \\
\hline Hyperlipidaemia & $16(0)$ & $88(0)$ \\
\hline Stroke & $13(0)$ & $87(0)$ \\
\hline Asthma & $406(8)$ & $180(0)$ \\
\hline Rhinitis & $1032(21)$ & $348(1)$ \\
\hline \multicolumn{3}{|c|}{ Age at $A D$ diagnosis (years) } \\
\hline $0-9$ & $998(21)$ & - \\
\hline $10-19$ & 798 (17) & - \\
\hline $20-29$ & 1272 (26) & - \\
\hline $30-39$ & 941 (20) & - \\
\hline $40-49$ & $556(12)$ & - \\
\hline $50-59$ & $222(5)$ & - \\
\hline $60-63$ & 27 (1) & - \\
\hline
\end{tabular}

the general population was 2.40 (95\% CI 1.27 to 4.54 ) among those in need of systemic treatment and 1.58 (95\% CI 1.02 to 2.45) among those not using systemic treatment.

No substantial variation was observed in the HRs according to gender, age at diagnosis, number of $\mathrm{AD}$ hospital contacts, and presence of asthma or rhinitis (table 2). Interactions between the exposure, $\mathrm{AD}$ and all covariates included in the models were tested and found statistically non-significant.

\section{DISCUSSION}

Hospital-diagnosed $\mathrm{AD}$ was associated with substantially increased MI risk compared with the general population.

The results of the present study are in line with existing reports of the increased prevalence of cardiovascular risk factors and history of self-reported heart attacks in individuals with $\mathrm{AD} .^{8}{ }^{11}$ Our findings extend the recent report on follow-up of patients with AD from 1997 and onwards, including adjustment for several indicators of cardiovascular risk factors in terms of medication use 
Figure 1 Risk of myocardial infarction in patients with atopic dermatitis and the

birth-year-matched and

gender-matched general population comparison cohort. $\mathrm{AD}$, atopic dermatitis; $\mathrm{MI}$, myocardial infarction.

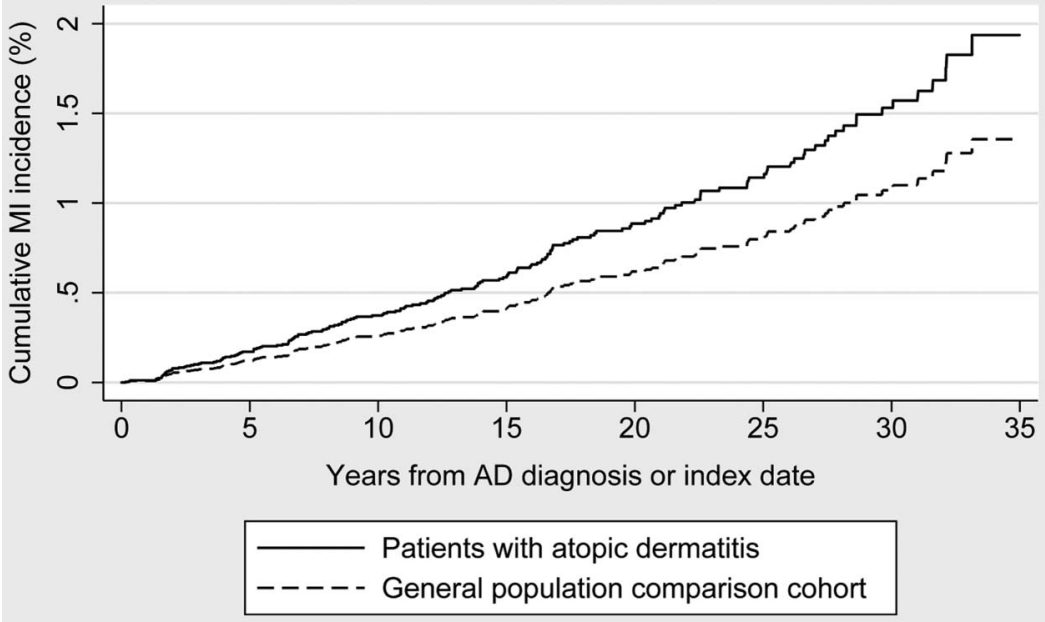

Table 2 HRs of myocardial infarction for individuals diagnosed with atopic dermatitis from 1977 to 1983 in Denmark, compared with a general population cohort matched for gender and birth-year

\begin{tabular}{|c|c|c|}
\hline \multirow[b]{2}{*}{ Characteristics } & \multicolumn{2}{|c|}{$\begin{array}{l}\text { Comparison with general population } \\
\text { cohort }\end{array}$} \\
\hline & $\begin{array}{l}\text { Crude HR } \\
(95 \% \mathrm{CI})\end{array}$ & $\begin{array}{l}\text { Adjusted HR } \\
(95 \% \mathrm{Cl})^{\star}\end{array}$ \\
\hline Overall & 1.79 (1.25 to 2.57$)$ & 1.74 (1.21 to 2.49$)$ \\
\hline Male & 2.03 (1.32 to 3.12$)$ & 2.01 (1.31 to 3.08$)$ \\
\hline Female & 1.39 (0.72 to 2.69$)$ & $1.28(0.66$ to 2.48$)$ \\
\hline \multicolumn{3}{|c|}{ Age at $A D$ diagnosis (years) } \\
\hline $0-19$ & $1.93(0.75$ to 5.01$)$ & 1.64 (0.62 to 4.33$)$ \\
\hline $20-$ & $1.76(1.20$ to 2.60$)$ & 1.76 (1.20 to 2.60$)$ \\
\hline \multicolumn{3}{|c|}{ Number of $A D$ hospital contacts } \\
\hline $2-4$ & 1.74 (1.13 to 2.67$)$ & 1.71 (1.11 to 2.64$)$ \\
\hline $5-9$ & $1.86(0.87$ to 3.95$)$ & 1.69 (0.79 to 3.63$)$ \\
\hline$\geq 10$ & 2.00 (0.58 to 6.92$)$ & $1.95(0.55$ to 6.91$)$ \\
\hline \multicolumn{3}{|c|}{ Use of systemic $A D$ treatment† } \\
\hline No & $1.62(1.04$ to 2.51$)$ & 1.58 (1.02 to 2.45$)$ \\
\hline Yes & 2.38 (1.26 to 4.50$)$ & $2.40(1.27$ to 4.54$)$ \\
\hline \multicolumn{3}{|c|}{ Asthma or rhinitis } \\
\hline No & 1.76 (1.17 to 2.64$)$ & 1.72 (1.14 to 2.59$)$ \\
\hline Yes & $1.97(0.92$ to 4.21$)$ & $1.73(0.80$ to 3.74$)$ \\
\hline \multicolumn{3}{|c|}{$\begin{array}{l}\text { *Adjusted for: birth-year categories, gender, educational level and } \\
\text { history of diabetes, hypertension, hyperlipidaemia or stroke. } \\
\text { †For the part ( } 4639 \text { patients with } A D) \text { of the study population still } \\
\text { at risk of incident MI by } 1995 \text {, when the prescription data were first } \\
\text { available. } \\
\text { AD, atopic dermatitis; MI, myocardial infarction. }\end{array}$} \\
\hline
\end{tabular}

and hospital diagnoses occurring both before and after AD diagnosis. ${ }^{12}$ To increase validity, we only included patients with $\mathrm{AD}$ with at least two separate hospital diagnoses of $\mathrm{AD}$, and we did sensitivity analyses to evaluate the validity of the $\mathrm{AD}$ diagnosis data. Furthermore, we followed patients with $\mathrm{AD}$ from as early as 1977 onwards for MI, immediately from the date of second AD diagnosis. This enabled evaluation of potential bias from follow-up of patients with prevalent rather than incident $\mathrm{AD}$ diagnoses. ${ }^{19}$ This was particularly relevant as we expected that a substantial proportion of the patients with $\mathrm{AD}$ were in fact prevalent patients with $\mathrm{AD}$ with $\mathrm{AD}$ since childhood, although they received an apparent incident, first time, hospital AD diagnosis as adults. This potential inclusion of patients with prevalent rather than incident $\mathrm{AD}$ will have left the HRs conservative, as those with MIs occurring before the $\mathrm{AD}$ diagnosis date were excluded. Subgroup analyses, although imprecise, indicated no substantial variation of the HR according to the age at $\mathrm{AD}$ hospital diagnosis. In the present study, we did not adjust for cardiovascular risk factors emerging after the $\mathrm{AD}$ diagnosis, in contrast to the study by Andersen $e t a l,{ }^{12}$ which may explain the stronger association between $\mathrm{AD}$ and $\mathrm{MI}$ of our study. However, it remains to be clarified, whether these cardiovascular risk factors are part of the pathophysiology of $\mathrm{AD}$, in which case, adjustment would lead to underestimation of the actual impact of $\mathrm{AD}$ on MI risk. Our findings are in accordance with those of our recent study of coronary artery disease in patients with severe $\mathrm{AD}$ assessed by cardiac CT angiography. These data showed an increased prevalence of coronary artery calcium in patients with $\mathrm{AD}$ than in controls (AD: $45.2 \%$, controls: $15.2 \%$; $\mathrm{p}=0.01$ ); and the contrast-enhanced CT angiography showed an increased prevalence of coronary segments with luminal stenosis among patients with $\mathrm{AD}$ (AD: $48.1 \%$, controls $21.2 \%$; $\mathrm{p}=0.03$ ). ${ }^{9}$

Potential mechanisms underlying the observed association include abnormal immunological pathways, which $\mathrm{AD}$ may have in common with psoriasis and other inflammatory disorders such as rheumatoid arthritis. ${ }^{20}$ However, factors other than inflammation, including psychosocial factors and increased prevalence of modifiable MI risk factors ${ }^{11}$ may also play a central role.

\section{Study strengths and limitations}

We had no data on AD diagnoses before 1977, and the maximum duration of follow-up was 35 years. Thus, it was not possible to follow patients with $\mathrm{AD}$ from early childhood to above 50 years of age, where MI would 
normally present. ${ }^{21}$ We therefore also included patients with $\mathrm{AD}$ diagnosed in adulthood. These patients may very well have had $\mathrm{AD}$ to a varying degree since childhood. This assumption is supported by a reported association between earlier onset of the disease in childhood and persistent $\mathrm{AD}$ in adulthood. ${ }^{6}$ We were able to perform analyses stratified on age at $\mathrm{AD}$ diagnosis, which indicated no variation of the HRs according to age at $\mathrm{AD}$ diagnosis. The virtually complete follow-up enabled by the CRS diminished selection bias due to loss to follow-up.

The validity of our estimates depends in particular on valid coding of $\mathrm{AD}$ and $\mathrm{MI}$ diagnoses. The data in the DNRP were reported to have a high positive predictive value for MI and several disease diagnoses. ${ }^{13}$ We restricted the $\mathrm{AD}$ cohort to patients with at least two hospital diagnoses of AD. The estimates did not change substantially after restriction of the $\mathrm{AD}$ cohort to patients with at least two hospital contacts with $\mathrm{AD}$ as the primary diagnosis. Furthermore, we would also expect a very high positive predictive value of the $\mathrm{AD}$ diagnosis in patients who filled prescriptions for cyclosporine, methotrexate or azathioprine. However, the lacking reports on the validity of ICD coding of the $\mathrm{AD}$ diagnosis in the DNRP is a limitation of this study.

When interpreting the adjusted HRs, it should be considered that the cardiovascular risk factors included in the models may actually be intermediate steps in a potential causal pathway from $\mathrm{AD}$ to $\mathrm{MI}$, and as such should not be adjusted for. In particular as a substantial proportion of the patients presenting at hospital with $\mathrm{AD}$ as adults may have had $\mathrm{AD}$ since childhood. We therefore also presented the crude estimates. We adjusted for MI risk factors in terms of history of diabetes, hypertension and hyperlipidaemia and we also adjusted for history of stroke, as a marker of increased thromboembolic risk. Furthermore, we were able to adjust for educational level. We did not have data on smoking, alcohol consumption and body mass index, and therefore we cannot rule out potential confounding from these MI risk factors. Data have indicated an increased prevalence of smokers in individuals with $\mathrm{AD}{ }^{11}$ Reports of mainly cross-sectional data suggest an increased prevalence of obesity in individuals with $\mathrm{AD}$ in some countries; however, it remains unclear if $\mathrm{AD}$ may lead to increased risk of obesity. ${ }^{22}$ Thus, obesity may also be an intermediate step in a pathway from $\mathrm{AD}$ to $\mathrm{MI}$, and as such should not be adjusted for in this study. ${ }^{23}$ We would expect a potential bias from obesity preceding $\mathrm{AD}$, in this Danish study population, to be less likely in the subanalysis including patients with $\mathrm{AD}$ diagnosed before age 20 years. In this subanalysis the HR did not differ substantially from that of patients 20 years or older at $\mathrm{AD}$ diagnosis. Furthermore, the cardiovascular risk factors that we were able to identify are all associated with obesity and therefore adjustment for these diseases will have mitigated any strong confounding potentially resulting from obesity.
We lacked detailed information on $\mathrm{AD}$ severity. A Danish study from 2005, indicated that during the 1980s and 1990s only about $2 \%$ of individuals with $\mathrm{AD}$ were hospitalised. ${ }^{24}$ We would expect the hospital diagnoses of patients with $\mathrm{AD}$ to have more severe $\mathrm{AD}$, and thus our findings may not be generalisable to individuals with mild $\mathrm{AD}$ treated by primary care physicians.

\section{Conclusion}

Hospital-diagnosed AD was associated with a substantially increased risk of MI compared with the general population. In the light of these results and the existing evidence of an increased prevalence of cardiovascular disease risk factors in the AD population, addressing modifiable cardiovascular disease risk factors as part of the standard medical follow-up of these patients, is recommended. The early onset of $\mathrm{AD}$, with most patients presenting during early childhood, may provide clinicians with a unique opportunity for promoting a heart healthy lifestyle in these young individuals and their families. However, the effects of preventive measures such as reduction of traditional risk factors for cardiovascular disease and optimal management of the dermatological aspects of $\mathrm{AD}$, remains to be clarified.

\section{Author affiliations}

${ }^{1}$ Department of Dermatology, Aarhus University Hospital, Aarhus, Denmark ${ }^{2}$ Department of Cardiology, Aarhus University Hospital, Aarhus, Denmark ${ }^{3}$ Department of Clinical Epidemiology, Aarhus University Hospital, Aarhus, Denmark

Contributors JLR made primary contribution to writing the manuscript. All authors contributed to the study conception and study design. MO performed data collection and statistical analyses and commented on the manuscript. All authors contributed to the interpretation of results, all revised the manuscript critically and all approved the final manuscript. MO is guarantor for this study.

Funding This study was supported by grants from the Aage Bang Foundation. The foundation played no role in the conduct of the research.

Competing interests None declared.

Provenance and peer review Not commissioned; externally peer reviewed.

Data sharing statement No additional data are available.

Open Access This is an Open Access article distributed in accordance with the Creative Commons Attribution Non Commercial (CC BY-NC 4.0) license, which permits others to distribute, remix, adapt, build upon this work noncommercially, and license their derivative works on different terms, provided the original work is properly cited and the use is non-commercial. See: http:// creativecommons.org/licenses/by-nc/4.0/

\section{REFERENCES}

1. Vestergaard C, Deleuran M. Advances in the diagnosis and therapeutic management of atopic dermatitis. Drugs 2014;74:757-69.

2. Schmitt J, Schmitt NM, Kirch W, et al. Outpatient care and medical treatment of children and adults with atopic eczema. J Dtsch Dermatol Ges 2009;7:345-51.

3. Paller AS, Chren MM. Out of the skin of babes: measuring the full impact of atopic dermatitis in infants and young children. J Invest Dermatol 2012;132:2494-6.

4. Worldwide variation in prevalence of symptoms of asthma, allergic rhinoconjunctivitis, and atopic eczema: ISAAC. The International Study of Asthma and Allergies in Childhood (ISAAC) Steering Committee. Lancet 1998;351:1225-32. 
5. DaVeiga SP. Epidemiology of atopic dermatitis: a review. Allergy Asthma Proc 2012;33:227-34.

6. Mortz CG, Andersen KE, Dellgren C, et al. Atopic dermatitis from adolescence to adulthood in the TOACS cohort: prevalence, persistence and comorbidities. Allergy 2015;70:836-45.

7. Gelfand JM, Neimann AL, Shin DB, et al. Risk of myocardial infarction in patients with psoriasis. JAMA 2006;296:1735-41.

8. Silverberg Jl, Becker L, Kwasny M, et al. Central obesity and high blood pressure in pediatric patients with atopic dermatitis. JAMA Dermatol 2015;151:144-52.

9. Hjuler KF, Böttcher M, Vestergaard C, et al. Increased prevalence of coronary artery disease in severe psoriasis and severe atopic dermatitis. Am J Med 2015;128:1325-34.e2.

10. Silverberg Jl. Association between adult atopic dermatitis, cardiovascular disease, and increased heart attacks in three population-based studies. Allergy 2015;70:1300-8.

11. Silverberg JI, Greenland P. Eczema and cardiovascular risk factors in 2 US adult population studies. J Allergy Clin Immunol 2015;135:721-8.e6.

12. Andersen $\mathrm{YM}$, Egeberg A, Gislason GH, et al. Risk of myocardial infarction, ischemic stroke, and cardiovascular death in patients with atopic dermatitis. J Allergy Clin Immunol 2016;138:310-312.e3.

13. Schmidt M, Schmidt SA, Sandegaard JL, et al. The Danish National Patient Registry: a review of content, data quality, and research potential. Clin Epidemiol 2015;7:449-90.

14. Pedersen CB, Gotzsche H, Moller JO, et al. The Danish Civil Registration System. A cohort of eight million persons. Dan Med Bull 2006;53:441-9.
15. Olsen M, Hjortdal VE, Mortensen LH, et al. Educational achievement among long-term survivors of congenital heart defects: a Danish population-based follow-up study. Cardiol Young 2011;21:197-203.

16. Olsen M, Sørensen HT, Hjortdal VE, et al. Congenital heart defects and developmental and other psychiatric disorders: a Danish nationwide cohort study. Circulation 2011;124:1706-12.

17. Kildemoes HW, Sorensen HT, Hallas J. The Danish National Prescription Registry. Scand J Public Health 2011;39(7 Suppl):38-41.

18. Gooley TA, Leisenring W, Crowley J, et al. Estimation of failure probabilities in the presence of competing risks: new representations of old estimators. Stat Med 1999;18:695-706.

19. Hernán MA. The hazards of hazard ratios. Epidemiology 2010;21:13-5

20. Moreira DM, da Silva RL, Vieira JL, et al. Role of vascular inflammation in coronary artery disease: potential of anti-inflammatory drugs in the prevention of atherothrombosis. Inflammation and anti-inflammatory drugs in coronary artery disease. Am J Cardiovasc Drugs 2015;15:1-11.

21. Bhatnagar $\mathrm{P}$, Wickramasinghe $\mathrm{K}$, Williams $\mathrm{J}$, et al. The epidemiology of cardiovascular disease in the UK 2014. Heart 2015;101:1182-9.

22. Zhang A, Silverberg Jl. Association of atopic dermatitis with being overweight and obese: a systematic review and metaanalysis. J Am Acad Dermatol 2015;72:606-16.e4.

23. Rothman KJ, Greenland S, Lash TL. Modern epidemiology. 3rd edn. Philadelphia: Lippincott Williams \& Wilkins, 2008.

24. Olesen AB, Bang K, Juul S, et al. Stable incidence of atopic dermatitis among children in Denmark during the 1990s. Acta Derm Venereol 2005;85:244-7. 